\title{
A cluster driven channel assignment mechanism for wireless mesh networks
}

\author{
Nicolas Letor and Chris Blondia \\ University of Antwerp - PATS - IBBT - Department of Mathematics and Computer Science \\ Middelheimlaan 1, B-2020 Antwerpen, Belgium \\ nicolas.letor@ua.ac.be \\ Stefan Bouckaert, Ingrid Moerman and Piet Demeester \\ Ghent University - IBCN - IBBT - Department of Information Technology (INTEC) \\ Gaston Crommenlaan 8/201, B-9050 Gent, Belgium \\ stefan.bouckaert@intec.ugent.be
}

\begin{abstract}
In this paper we present a cross layer topology preserving channel selection protocol for a two interface wireless mesh network. The presented protocol is a cluster driven channel selection protocol, preserving a single interface to maintain connectivity and using the other interface to participate in small clusters. The clusters are organized by an independent set clustering algorithm, in which the cluster head dictates the operating channel for the local cluster. We show that the channel selection algorithm doubles the capacity for both a regular grid and a random topology.
\end{abstract}

\section{Introduction}

Wireless mesh networks have become increasingly popular, which can be attributed to their low cost and their auto organizing features. For example wireless mesh networks have been deployed to provide internet access to remote sites [15] and urban areas [20]. Other projects like [4] take advantage of the auto organizing feature to create an instantaneous infrastructure for emergency services, providing video and communication services.

The main disadvantage of a wireless mesh network is the limited capacity and scalability of these networks [8]. To remediate this capacity problem, researchers have been investigating the use of multi-interface equipped mesh nodes. These multiple interfaces have to be coordinated to operate on different channels, creating the need for a channel selection algorithm. In this paper we present a cross-layer channel selection mechanism as a solution for this capacity problem.

This paper is further organized as follows: in the next section we will discuss related work on channel selection.
In section 3 we explain our design goals and requirements, which led to the architecture of the proposed channel assignment protocol. In section 4 we evaluate the proposed solution, we describe some future work in section 5 and we conclude this paper in section 6 .

\section{Related work}

Several channel selections mechanisms have been proposed in literature. Depending on how these channels assignments are computed, they can be classified into three types: link-channel negotiation protocols, global network organization mechanisms and distributed channel assignment techniques.

Some protocols select a channel by performing a link negotiation protocol to determine a suitable channel. The authors of $[9,13]$ presented a modified 802.11 MAC protocol, which allowed a channel to be negotiated in the RTS/CTS exchange. After the negotiation phase, both participating stations switch to the agreed channel to perform the data transmission. Another solution is to virtualize multiple wireless interfaces into a single interface and to select the most suitable interface for transmission [1]. However previous solutions are completely integrated in the link layer, which means they cannot take advantage of higher layer information like network topology or the channel assignment of nearby interfering links.

Other researchers $[17,18,21]$ consider the channel selection problem as a network organization problem, in which they transpose the channel assignment problem to a graph coloring problem. The disadvantage of such a solution is that the channel assignment computation has to be centralized on a single entity. As a consequence, the central entity must be notified of the complete network status status. Not only does this result in a single point of failure, it also re- 
quires control packets to be transmitted to the central point, resulting in large overhead traffic.

Distributed channel selection algorithms avoid the single point of failure by optimizing the channel assignment in the local neighborhood of mobile station. In [5] the authors proposed an on demand channel assignment protocol, that assigns links to channels when these links become active. When a new flow is forwarded over a not used link, it assigns the activated link to a wireless interface, corresponding to a wireless channel. The channel is selected using a channel quality variable, representing the channel occupancy in the local neighborhood. In [10] a self-stabilizing greedy channel selection algorithm is proposed, which allows each node to select the least interfered channel for a wireless interface.

The authors of [23] took a different approach (based on the work of [7] for TDMA networks) by organizing the network in small clusters, where each individual cluster communicates on its own selected channel. The clusterhead is responsible for selecting a suitable channel and communicating the selected channel to the cluster members. Our proposed channel selection shares the same basic ideas with the previous technique, but instead of developing a pure L2 solution, we opted for a cross layer channel selection protocol integrated with the mesh routing protocol. This integration enables the channel selection technique to access the network topology information in order to optimize the clustering (section 3.1) and it enables a cluster(head) to intelligently select a channel in agreement with other clusters (section 3.2). Another advantage is that single interface (mobile) stations can participate in the network (section 3.3).

\section{Cluster driven channel selection}

The authors of [2] noted that there are two types of nodes participating in a wireless mesh network: backbone routers and mobile devices. Usually the former type of nodes are equipped with multiple wireless interfaces, while the latter type of nodes only have a single interface. Therefore we required that a channel selection protocol should be backwards compatible with single interface devices. This implies that the channel selection protocol should be a topology preserving solution, unless a separate wireless interface is provided for the mobile clients. Otherwise a channel selection solution could disconnect a mobile station, resulting in a partitioned wireless mesh network.

Our goal was to develop a protocol which could be implemented using standard COTS 802.11 equipment hardware. The authors of [19] showed that is not feasible to develop a solution with more than 2 interfaces. Therefore we were forced to develop a solution using only two interfaces, as to avoid any inter radio interference, caused by

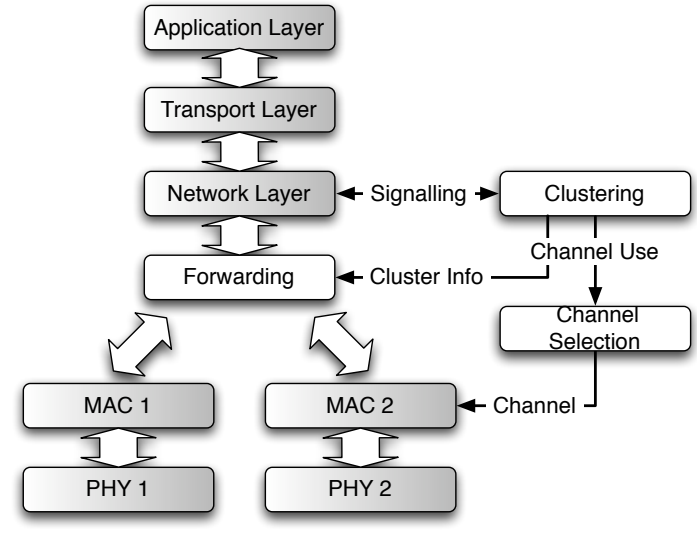

Figure 1. Architecture

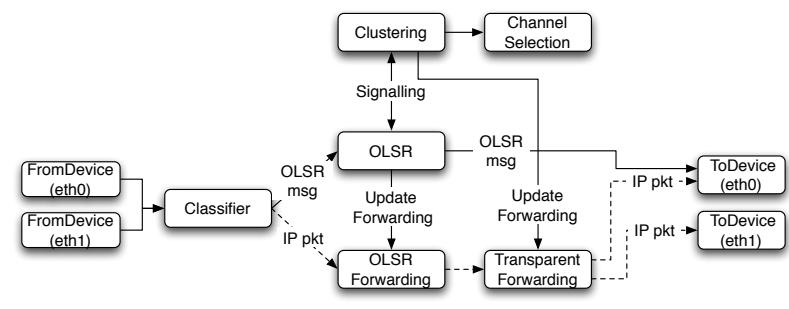

Figure 2. OLSR integration

insufficient shielding of these devices.

We satisfy both requirements by configuring one interface to a common fixed channel to maintain connectivity allowing single interface (mobile) nodes to participate in the network - and using the other remaining interface to partition the wireless mesh network in small cells, each operating on a separate channel. This approach mimics the behavior of 802.11 infra-structured cells, each running on a dedicated channel.

Our solution comprise three modules, shown in figure 1. Firstly the clustering component is responsible for creating a local group and selecting a channel coordinator (section 3.1). Secondly the channel selection component enables the channel coordinator to select a suitable channel for use in the local cluster group (section 3.2). Finally the forwarding module is responsible for transmitting the packet using the most suitable wireless interface (section 3.3). These three components were integrated in the Optimized Link State Routing (OLSR) [6] protocol (figure 2), which is a well known and widely used routing protocol for wireless mesh networks. The integration allows each channel selection component to retrieve and store information in the OLSR information databases, eliminating the need to maintain separate information databases. 


\subsection{Clustering module}

The clustering module is responsible for organizing the network in small distinct cells, coordinated by a channel coordinator. As a consequence it is undesirable that two channel coordinators are within communication range. We reformulated this requirement into an Independent Set problem. An Independent Set of a graph is a subset of the vertices such that any two vertices, member of this independent set, are not directly connected. In this case an independent set means that each channel coordinator, as a member of this independent set, will not be in communication range with another channel coordinator.

The authors of [3] proposed a message-driven Maximal Weighted Independent Set algorithm for a network graph, where each node has an associated weight parameter. We translated this algorithm into a soft-state independent set clustering (ISC) algorithm (shown in Algorithm 1) to avoid any synchronization issues due to the simultaneous state changes. For example, if a new clusterhead advertises itself, all neighboring stations may decide to join this new cluster. These stations will simultaneously send update messages, resulting in collision of broadcast packets on the MAC layer. Transforming this algorithm to a soft-state version, where each station periodically advertises its status avoids this synchronization problem.

A crucial parameter in this algorithm is the weight variable, associated with each node. We explored three possible heuristics for assigning a weight to a mesh node. The first possibility is a fixed external importance value, which we simulated by assigning a random value to each node, leading to a random ISC channel selection (ISC-R). A second possibility is to represent the degree of connectivity [7], in which the highest connected node will become channel coordinator (ISC-H). And finally we explored a metric representing the distance (in hops) to the center (ISC-C). Figure 3 shows a possible clustering applied to a grid topology for each of these three heuristics.

The clustering module is implemented as an OLSR plugin (figure 2), which piggybacks the cluster advertisement on the HELLO advertisements of the routing protocol. By piggybacking the signaling packets unnecessary contention for the wireless medium is avoided. Each clustering message contains the following information: a weight parameter indicating its importance, the membership status (clusterhead, cluster member or no member), clusterhead IP address and the channel of the cluster. When the clustering module receives a cluster advertisement message, it will analyze the membership status of the originating station and store this information in the OLSR neighbor database, where it can be accessed by the forwarding module. The channel is reported to the Channel Selection module, informing it of neighboring cluster's channel usage.

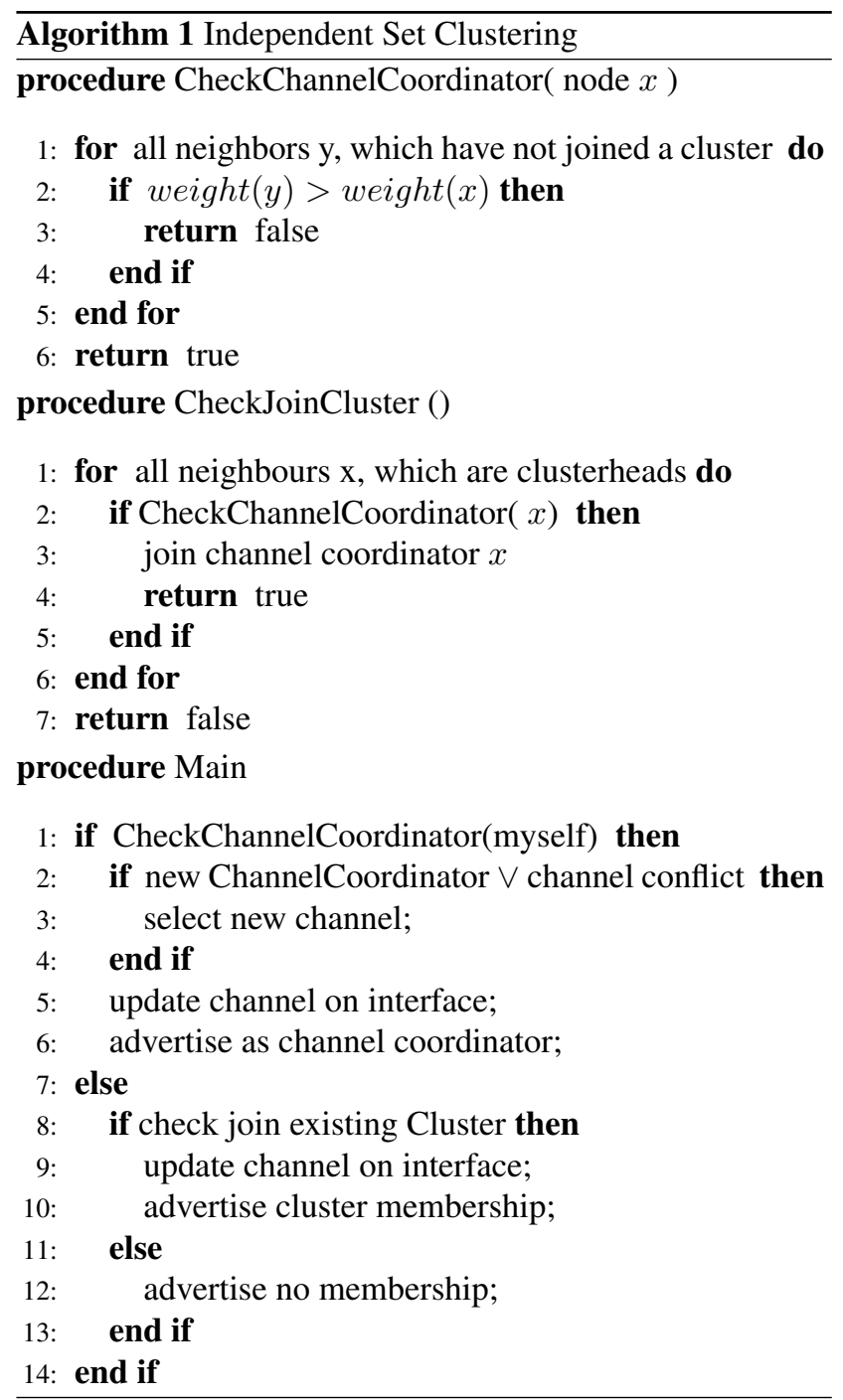

\subsection{Channel assignment}

The previous section shows how the network is organized in clusters and how the different clusters are coordinated to use a common channel, dictated by the channel coordinator. But how does the channel coordinator select the optimal channel? The choice of a suitable channel is made by the Channel Assignment module. This module is kept informed by the clustering module about the channel usage of the neighboring clusters. When a new channel is requested, it will compute a frequency map $F(i)$ for each channel, denoting how many clusters are operating on channel $i$. Based on this frequency map, it computes a Channel Quality Metric (CQM), indicating how much interference the cluster will receive from neighboring clusters operating on nearby channels. 


$$
\begin{aligned}
C Q M_{i}= & \sum_{|i-j|<3} C P(i, j) * F(j) \\
C P(i, j) & =4 \Leftarrow|i-j|=0 \\
& =1 \Leftarrow|i-j|<3 \\
& =0 \Leftarrow|i-j| \geq 3
\end{aligned}
$$

The channel assignment module will select the channel with lowest associated CQM value. The CQM value assumes that the wireless interface is operating in the $2.4 \mathrm{Ghz}$ frequency band, where channels are not orthogonal. The channel proximity function $C P(i, j)$ attenuates the interference of nearby channels.

\subsection{Transparent forwarding}

The purpose of the forwarding component is to select on which interface (the default interface or the cluster interface) a data packet will be transmitted. It received this data packet from the OLSR forwarding module, which has determined to which next hop mesh node this packet should be transmitted. The forwarding component retrieves the cluster membership information associated with this next hop in the neighbor information database. If the transmitting node and the next hop are member of the same cluster, then the module will select the second interface, offloading this data transmission from the common channel to the cluster channel. Otherwise the packet will be forwarded using the standard mesh interface on the common channel, because the two stations are not members of the same cluster or because no membership information available, suggesting that the next hop has only a single interface.

We note that the design of the channel selection technique is transparent to the OLSR routing protocol. The technique accesses the routing protocol information, but does not interfere with the routing operation, i.e. the path calculations, allowing a standard single interface (OLSR) node to participate in the mesh network.

\section{Simulation study}

The proposed cluster driven channel assignment algorithm has been implemented using the Click Modular Router software [11]. Click allows to program and modify networking protocols in a fast and configurable way. The performance analysis of the Click configurations were performed in the Nsclick [16] simulator, extended with wireless cross-layer design features [12]. These features allow the Click router to have complete control over 802.11 wireless interfaces, allowing the transmission of raw 802.11 data

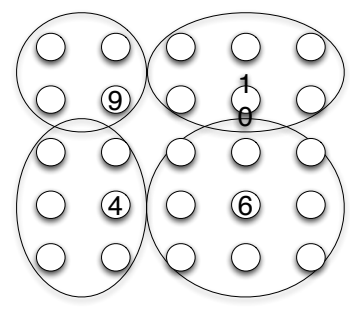

(a) ISC-H clustering

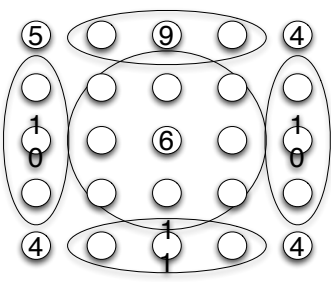

(b) ISC-C clustering

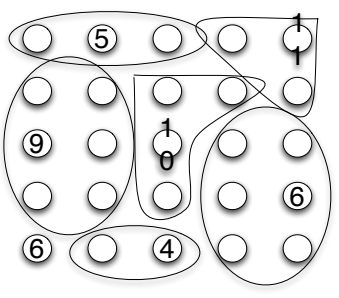

(c) ISC-R clustering

Figure 3. 5x5 Grid scenario

frames, secondly the retrieval of transmission information and finally the possibility to change the wireless channel from within the software router. These features are supported in wireless hardware, using the Madwifi driver [14].

Using the Click Modular Router as a platform, we compared our solution against a single interface mesh network and a two interface reference mesh network as a benchmark. This reference mesh network is an a priori channel assigned network, which is organized in a tree like structure from the geographical center of the network. The channel assignment in this network was constructed in two steps. First an interference conflict graph is constructed. A vertex coloring [22] is performed on this conflict graph, creating a noninterfering channel assignment for each station's secondary interface. In the second step the network is connected using a Breadth First Search (BFS) graph traversal algorithm, originating from the center of the network, where each primary interface of a node is connected with the secondary interface of its parent. The purpose of this channel assignment mechanism is to have an indication on how much a network can be improved in the optimal case i.e. if a solution is not required to preserve the topology of the original network.

Grid topology The first network we have simulated is a five by five grid topology (figure 3 ) with a $175 \mathrm{~m}$ inter node distance. The transmission rate was fixed at $2 \mathrm{Mbps}$ to study the effects of the channel selection on the medium capacity. The transmission range was set to $250 \mathrm{~m}$ and the carrier sensing range was set to $550 \mathrm{~m}$. The network warmup time was 30 s and traffic was generated during 120 s. The results 


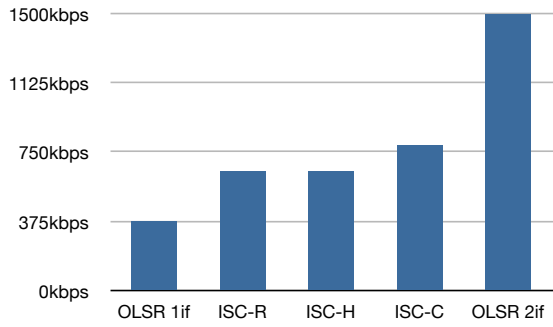

(a) Relay capacity

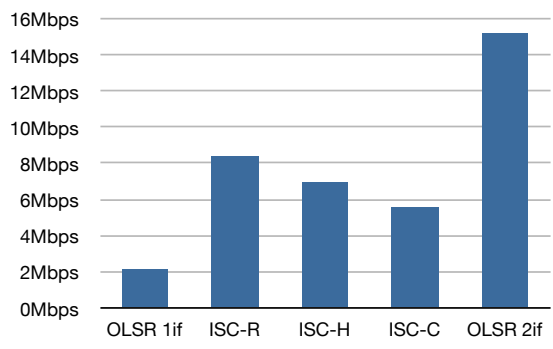

(b) Aggregated network capacity

Figure 4. 5x5 Grid scenario

are averaged over 25 runs. The OLSR routing protocol parameters are summarized below.

\begin{tabular}{lr}
\hline Parameter & Value \\
\hline HELLO_INTERVAL & $1 \mathrm{~s}$ \\
TC_INTERVAL & $5 \mathrm{~s}$ \\
NEIGHBOR_HOLD_TIME & $6 \mathrm{~s}$ \\
TOP_HOLD_TIME & $15 \mathrm{~s}$ \\
\hline
\end{tabular}

In order to measure the maximum capacity of the mesh network, it has to be saturated with traffic. However in a saturated wireless mesh network, the broadcast HELLO messages are easily lost as they are not retransmitted, resulting in false notifications of link failures. Note that we doubled the rate at which these HELLO messages are sent in order to compensate for this effect.

We studied the effects of the channel selection technique on the intra path interference by measuring the relaying capacity. This relaying capacity was measured by sending a CBR stream along the longest path in the network and monitoring if the network could sustain the CBR stream. The highest sustainable CBR stream along this path was recorded as the relaying capacity. Figure 4(a) shows that the ISC-R cluster driven channel selection manages to double the relaying capacity, indicating that the intra path interference is reduced by half. The other variants variants (ISC-H and ISC-R) are not able to exploit the regular and symmetrical properties of the grid topology. They only show a performance gain of 70 percent. The reference channel selection

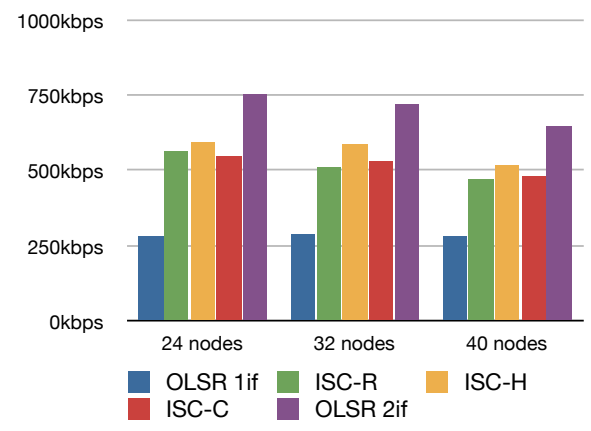

(a) Relay capacity

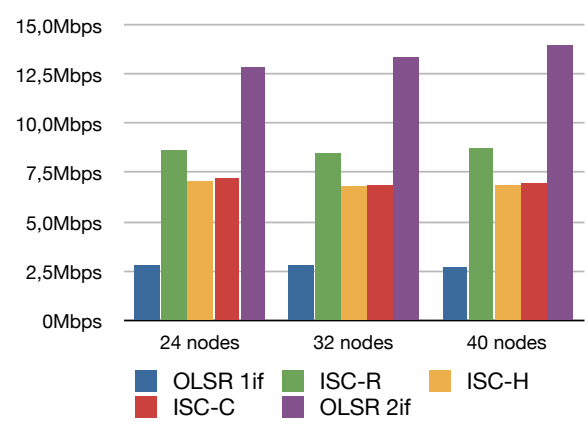

(b) Aggregated network capacity

Figure 5. Random topology scenario

outperforms the other channel selections and quadruples the available capacity for long distance relaying.

The effects of the channel selection protocol on the inter path interference were studied by measuring the aggregated network capacity. A random UDP stream, with a packet size of 1000 bytes, was generated at each mesh node directed to the local neighborhood of the generating node. The average throughput of all transmitting stations was recorded as the aggregated network capacity. Figure 4(b) shows that the cluster driven channel selection algorithms triple the capacity of the network, however they are outperformed by the reference channel selection with a factor of two.

The non topology preserving property of the channel assignment in the reference network gives a significant performance advantage compared to the cluster driven topology preserving channel selection algorithms. This advantage allows the channel selection to divide a local connected group of mesh nodes over different channels, reducing medium contention as there are less neighbors contending for channel access, while increasing capacity, as the local connected group exploits multiple channels in parallel. 


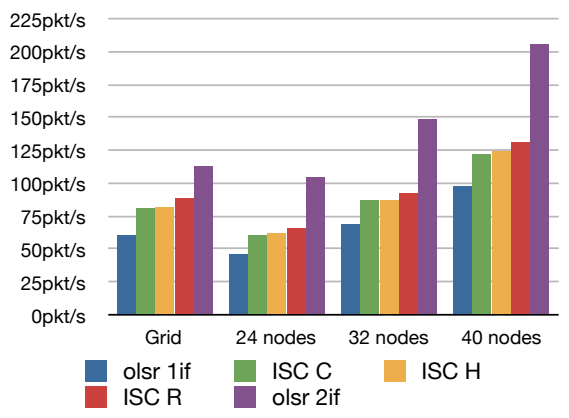

(a) Average \# packets transmitted per second

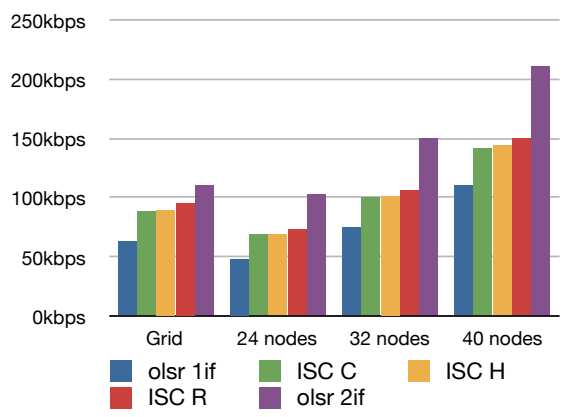

(b) Average \# bytes transmitted per second

\section{Figure 6. Signaling Overhead}

Random topology As a second case study we analyzed the performance of the channel selection protocol in a network with 24, 32 and 40 nodes, placed randomly in rectangular area of $1200 \mathrm{~m}$ by $400 \mathrm{~m}$. Of each type 50 different topologies were generated and analyzed. The simulation parameters were kept the same as in the previous case study.

Figure 5(a) shows that all cluster driven channel selections techniques double the relaying capacity in comparison with a single interface mesh network, indicating that the intra path interference has been halved by the cluster driven channel selection algorithm. The reference channel selection outperforms the cluster driven channel selection mechanisms, tripling the relaying capacity compared to the single interface mesh network.

Figure 5(b) shows that the network capacity can be significantly improved. The cluster driven channel selection algorithms enhance the capacity by a factor of more than two. As in the previous scenario, the reference channel selection outperforms the other cluster driven channel selection algorithms by a factor two.

Figure 6 shows the signaling overhead for both the grid and random topologies. The presented data indicate that the cluster driven channel selection algorithms increase the signaling load significantly compared to the OLSR protocol on a single interface network. However, when comparing this increase to the two-fold increase for OLSR running on both interfaces of a mesh node, it can be seen that the cluster driven channel selection algorithms cause $25 \%$ less overhead.

The performance studies of the grid topology and the random topologies indicate that our channel selection algorithm consistently improves the capacity of a two interface wireless mesh network. If we compare this algorithm against the reference network channel selection, we conclude that relaxing the topology preservation requirement can deliver a huge performance bonus for the network capacity.

\section{Future work}

Currently we did not consider mobile nodes in our performance evaluation as we only performed a simulation study to determine the overall performance capacity increase in the wireless mesh backbone. We will study the impact of the channel assignment on the network performance perceived by the mobile nodes.

Secondly we will investigate the use of a traffic aware metric. A traffic aware metric could optimize the capacity of the network, by dynamically adapting the weight assignments on each backbone mesh router to reflect the current traffic conditions. The network will react by reorganizing the cluster grouping, and as a consequence it will also reorganize the channel assignment in the wireless mesh network. This could enable the mesh backbone routers to offload more traffic to their secondary interfaces in locations where a high bandwidth is demanded.

\section{Conclusion}

In this paper we have proven that a channel selection algorithm can improve the capacity of a wireless mesh network significantly. We have presented a distributed cross layer cluster driven channel selection algorithm which performs a channel selection in two interface wireless mesh networks and which preserves the topology of the network. This channel selection algorithm doubles the capacity of the network.

If a wireless mesh network is not expected to have mobile nodes or to be compatible with single interface nodes, then the topology preserving constraint can be removed. In this case we have shown that the capacity of a network can be improved even further, quadrupling the capacity of the mesh network, justifying the need for dedicated channel selection algorithms in wireless backhaul networks. 


\section{References}

[1] A. Adya, P. Bahl, J. Padhye, A. Wolman, and L. Zhou. A multi-radio unification protocol for ieee 802.11 wireless networks. In BROADNETS '04: Proceedings of the First International Conference on Broadband Networks (BROADNETS'04), pages 344-354, Washington, DC, USA, 2004. IEEE Computer Society.

[2] I. Akyildiz, X. Wang, and W. Wang. Wireless mesh networks: A survey, 2005.

[3] S. Basagni. Finding a maximal weighted independent set in wireless networks. Telecommunication Systems, 18(13):155-168, September 2001.

[4] J. Bergs, D. Naudts, C. Blondia, N. V. den Wijngaert, I. Moerman, and P. Demeester. A wireless network for emergency services: a multi-channel ad-hoc approach. In Proc. The Wireless Rural and Emergency Communications Conference, OCT 2007.

[5] S. Bouckaert, N. Letor, C. Blondia, I. Moerman, and P. Demeester. Distributed on demand channel selection in multi channel, multi interface wireless mesh networks. November 2007.

[6] T. Clausen and P. Jacquet. RFC 3626: Optimized Link State Routing Protocol (OLSR), October 2003.

[7] M. Gerla and J. Tsai. Multicluster, mobile, multimedia radio network, 1995.

[8] P. Gupta and P. R. Kumar. The capacity of wireless networks. IEEE Transactions on Information Theory, 46(2):388-404, 2000.

[9] N. Jain, S. Das, and A. Nasipuri. A multichannel csma mac protocol with receiver-based channel selection for multihop wireless networks, 2001.

[10] B. Ko, V. Misra, J. Padhye, and D. Rubenstein. Distributed channel assignment in multi-radio 802.11 mesh networks, 2007.

[11] E. Kohler, R. Morris, B. Chen, J. Jannotti, and M. F. Kaashoek. The Click Modular Router. ACM Transactions on Computer Systems, pages 263-294, August 2000.
[12] N. Letor, P. de Cleyn, and C. Blondia. Enabling cross layer design: adding the madwifi extensions to nsclick. In $A c$ cepted at First International Workshop on Network Simulation Tools 2007 in conjunction with Valuetools 2007, October 2007.

[13] J. Li, Z. J. Haas, M. Sheng, and Y. Chen. Performance evaluation of modified ieee 802.11 mac for multi-channel multi-hop ad hoc network. In AINA '03: Proceedings of the 17th International Conference on Advanced Information Networking and Applications, pages 312-317, Xi'an, China, 2003. IEEE Computer Society.

[14] Multiband Atheros Driver for WiFi. http://sourceforge.net/projects/madwifi/.

[15] Nepal wireless project. http://www.nepalwireless.net/.

[16] M. Neufeld, A. Jain, and D. Grunwald. Nsclick: Bridging Network Simulation and Deployment. In Proceedings of the 5th ACM international workshop on Modeling analysis and simulation of wireless and mobile systems, pages 74-81. ACM Press, 2002.

[17] K. N. Ramachandran, E. M. Belding-Royer, K. C. Almeroth, and M. M. Buddhikot. Interference-aware channel assignment in multi-radio wireless mesh networks. In INFOCOM. IEEE, 2006.

[18] A. Raniwala, K. Gopalan, and T. cker Chiueh. Centralized algorithms for multi-channel wireless mesh networks, 2004.

[19] J. Robinson, K. Papagiannaki, C. Diot, X. Guo, and L. Krishnamurthy. Experimenting with a multi-radio mesh networking testbed. In Proceedings of the First Workshop on Wireless Network Measurements, April 2005.

[20] Seattle wireless. http://www.seattlewireless.net/.

[21] A. P. Subramanian, R. Krishnan, S. Das, and H. Gupta. Minimum interference channel assignment in multi-radio wireless mesh networks, 2005.

[22] G. Szekeres and H. S. Wilf. An inequality for the chromatic number of graph. Combinatorial Theory, 4(1), January 1968.

[23] J. Zhu and S. Roy. 802.11 mesh networks with two-radio access points. In 2005 IEEE International Conference on Communications, volume 5, pages 3609 - 3615, May 2005. 